\title{
Cognitive Behavior Family Therapy of Psychosomatic Disorders with an Emphasis on Positive Psychotherapy
}

\author{
Mohammad Khodayarifard, Saeed Akbari Zardkhaneh \\ Psychotherapy \& Child Clinical Psychologist, Faculty of Psychology and Education, \\ University of Tehran, Tehran, Iran \\ Email: khodayar@ut.ac.ir, akbari76ir@yahoo.com
}

Received August $15^{\text {th }}, 2012$; revised September $17^{\text {th }}, 2012$; accepted October $14^{\text {th }}, 2012$

\begin{abstract}
Objective: The purpose of the present study is to examine the effectiveness of cognitive behavioral family therapy with an emphasis on positive psychotherapy in treating psychosomatic disorders. Method: A single subject design was applied. Three patients suffering from chronic lower back and neck pain were selected as a sample group and participated in 16 sessions of family therapy, two hours per week. Examination by a physician, X-ray and clinical interview based on DSM IV-TR along with MMPI and SCL90-R tests were used as instruments. Result: Almost all the subscales of MMPI and SCL-90-R and other instruments showed significant decrease after therapy as compared with the pretest results. Discussion: The results indicated the effectiveness of cognitive behavior family therapy based on positive psychotherapy. Some possible reasons could be solving conflicts and interpersonal problems by means of training certain skills such as problem solving, developing relationships with others, effective coping, assertiveness and positive thinking.
\end{abstract}

Keywords: Positive Psychotherapy; Psychosomatic Disorders; Family Therapy; Cognitive Behavior Therapy

\section{Introduction}

The relationship between body and mind has always been a very controversial issue. Human beings are viewed as a biopsychosocial organism and these three aspects affect each other interactional. This standpoint has brought about new interdisciplinary fields in psychology and medicine and these kinds of treatments are widely applied for treating psychosomatic disorders.

Psychosomatic disorders are psycho physiological disorders and include physiochemical, anatomical or physiological disorders (Knapp, 1985). Psychosomatic disorders like asthma, stomach ulcer, high blood pressure, muscular and bone pains and headache constitute a group of physical disorders which are caused by psychological problems or are at least intensified under the influence of psychological stressors.

Today there is a dominant view that almost all the physical diseases are potentially related to psychological factors. According to DSM-IV-TR (2000), in case of any kind of psychological disorders, identification of stressors and other psychological factors that amplify the symptoms is essential for the diagnosis of psychosomatic disorders. This group of disorders implicates the presence of a reciprocal and meaningful organic relationship between psychological and physical aspects of human beings.

Findings of many controlled studies have shown that the prevalence rate of psychosomatic disorders in industrial societies has been several times more than that in underdeveloped and traditional societies (Wolman, 1988). Mison (1975) believed that daily stressful experiences and living in big cities and industrial societies can be the cause of a change in the endocrine glands. This change can in turn affect the functions of the immune system and increase the vulnerability of an organism to disease. Furthermore, stressful events can affect the biological function of amino-acids in the central nervous system, prompt the decrease in the resistance of human and, conesquently, lead to psychosomatic disorders. Considering the role of different environmental, cognitive and bio-chemical factors that cause psychosomatic disorders, their treatment may also require the combination of several professional fields.

Today, there are several approaches for ameliorating psychosomatic disorders which include medical (e.g. surgical, pharmacological) and psychological (e.g. psychoanalytical, behavioral, cognitive and cognitive behavioral) approaches. However, it is not possible to decide with certainty about the type of therapy method or approach which is optimal for each problem. Although research has shown the efficacy of pharmacotherapy in decreasing the symptoms of psychosomatic problems related to spinal column, joints and muscles (Woodham, 2000), treatment of psychosomatic disorders has increasingly focused on the interaction of organic and psychological factors (Lipowski, 1985). In fact, the aim of treatments for psychosomatic disorders is not only to relieve the symptoms but also to prevent from their relapse. In treating the primary psychological factors which have a role in causing the somatic symptoms, psychotherapy can help beyond the efficacy of pharmacological treatments.

Applying cognitive behavioral techniques, whether in the form of individual or family therapy, is very common in treating psychosomatic disorders. Jay et al. (1987) compared cognitive behavioral family therapy, use of medicine and attentioncontrol techniques in relieving marrow bone pain. The results showed that cognitive behavioral family therapy was the most efficient method in decreasing this pain. It has been also 
observed that family therapy based on cognitive behavioral techniques can be effective in treating children who have psychosomatic disorders. Leibman et al. (1974) used cognitive behavioral techniques for treating a group of children who had severe asthma. In this psychosomatic disorder, the primary allergic tendency in the patient can be combined with emotional factors and, particularly with family problems and conflicts.

The members of a family are all elements of an interactive system in which one individual constantly influences and is influenced by all other members (Stierlin, 1977). Therefore, a symptom can be only understood within the framework and web of relationships in which that symptom is expressed. This is especially true for psychic and psychosomatic illnesses. The role that the symptom plays within the family, within its rules of conduct and within its social context can be only understood considering this viewpoint (Peseschkian, 1986).

Cognitive behavioral family therapy aims at reducing the symptoms as well as changing the dysfunctional family structure and roles through cognitive behavioral approach. This approach consists of different techniques such as training communication skills, problem solving, relaxation and rewarding system management.

Psychosomatic disorders (muscular/joint) are local reflections of anxiety, tension or other emotions in an individual as a muscular tonus. Anxiety plays an important role in the cognition, affection and behavior of the patients who have chronic muscular/joint pains (Asmundson, 1994). Among the researches who have studied the efficiency of cognitive behavioral family therapy in the treatment of psychosomatic muscular-joint pains, the following examples can be pointed out:

Kendal et al. (1997) treated a group of individuals suffering from anxiety disorders and muscular pains using a cognitive behavioral approach. In this research, the cognitive components of anxiety (such as recognizing physiological symptoms of anxiety, correcting stressful conversations, compromising different stressful situations and self-monitoring) were applied to the experimental group during 16 weeks. They were also trained with some behavioral techniques (like modeling, desensitization, role playing and relaxation) in order to control anxiety. At the end of the treatment period, anxiety symptoms were significantly reduced in the experimental group as compared with the control group.

Kazdin and Weisz (1998) compared individual and family cognitive behavioral therapy in the reduction of psychosomatic symptoms. The results for individual and family cognitive behavioral therapy were $57.1 \%$ and $84 \%$ improvement, respectively. Johnson and Dahl (1998) investigated the effects of a cognitive behavioral pain control program in a group of participants with muscular bone pains in two groups: the control group (study 1) and a sample of patients (study 2) that were studied during a long period. The four-week therapy programs included instructional sessions, explaining objectives, nerve calming, practice of social skills, reduction of the use of medicines and reversion to work programs.

Clinicians have been interested in applying different methods of psychotherapy including behavior therapy (Kazdin \& Wesiz, 1998) and positive family therapy (Peseschkian, 1986) in treating psychosomatic disorders and also investigating the efficiency of cognitive behavioral techniques in the treatment of muscular and bone disorders (Johnson \& Dahl, 1998). The main goal of the present research was to study the effect of cognitive behavioral family therapy on the treatment of psy- chosomatic disorders (muscular and bone pains).

\section{Method}

The present research was a single-subject study using an A-B design. In order to choose the research sample, 3 individuals (2 male and 1 female) with muscular-bone type of psychosomatic disorders were chosen from individuals who referred to a private psychotherapy clinic. However, since two of these participants (1 man and 1 woman) did not continue to participate in all of their therapy sessions, they were eliminated from the original sample and the sample was eventually consisted of three remaining participants.

All of the participants were diagnosed with psychosomatic disorder like muscular-bone problems (backache-neck pain) without physiological sources. The diagnosis was confirmed by $\mathrm{X}$-ray, clinical interview made by psychotherapist based on DSM-IV-TR, SCL-90-R and MMPI-2 testes. Data collection was conducted in two stages of pretest (stage A) and posttest (stage B).

Minnesota Multiphase Personality Inventory (MMPI) has 13 subscales, 3 subscales of which are related to test validity (L, F and $\mathrm{K}$ ) and other 10 subscales of Hypochondria (Hs), Depression (D), Hysteria (Hy), Psychosocial Deviation (Pd), Masculinity-Feminity (MF), Paranoia (P), Psychasthenia (Pt), Schizophrenia (Sc), Hypomania (Ma) and Social Introversion (Si) are related to personality and clinical indexes. The method of answering these questions is true/false; each true answer receives 1 score while the false answer receives 0 .

The validity of MMPI has been confirmed. The reliability of all MMPI subscales was also confirmed in a meta-analysis study and the range of reliability coefficients was reported between 0.71 for Ma scale and 0.84 for Pt scale (Marnat, 1990). In the present research, the short form of MMPI was used which included 71 questions. The participants' scores in Hs scale (or hypochondria) were considered as an index of their physical problems.

The Symptom Checklist-90-Revised (SCL-90-R) is a diagnostic and screening test used mainly for mental patients and drug addicts and alcoholics (Mirzaei, 1980). It consists of 90 items and 9 dimensions which measure different psychological aspects including somatization, obsessive-compulsive traits, interpersonal sensitivity, depression, anxiety, hostility, phobia, paranoia and psychoticism. By applying Cronbach's alpha coefficient, Mirzaei (1980) reported the reliability of the SCL$90-\mathrm{R}$ as 0.70 and its concurrent validity with the MMPI-2 was 0.51. According to Hooman (1997), the internal consistency coefficients of this instrument's subscales, which ranged from 0.69 to 0.88 , were acceptable. The construct validity and concurrent validity of this instrument were also acceptable according to Hooman (1997) and Nadjarian and Davoodi (2001). In the present study, the Cronbach's alpha coefficient was 0.97 for the whole test which ranged from 0.74 to 0.87 in the subtests. The correlation between the two halves of the test was 0.89 . In this research, the participants' score in the subscale of physical complaints was considered as the index of their physical problems.

\section{Procedure}

As mentioned before, the research sample included 3 individuals diagnosed with muscular bone psychosomatic disorder. 
They participated in 16 sessions of family therapy, $2 \mathrm{~h}$ per week. The techniques used in these sessions included training skills such as positive thinking, behavior analysis, and problem solving, anger control, and communication, assertiveness, coping with stress, interpersonal coping, relaxation and cognitive restructuring. Some of these techniques were cognitive and some of them were behavioral.

Cognitive restructuring through positive thinking is used in the family context to treat the client's depression and enhance the family members' relationships. Positive thinking is a cognitive behavioral technique that has been effective as a treatment method for many disorders (Khodayarifard, 2000). Training positive thinking is based on focusing on one's own and others' points of strength. This technique is performed in eight steps and may be applied to families or groups:

1) Each person writes all of his/her own positive points on a sheet of paper.

2) Each person thinks about at least five of his/her good experiences or memories and writes down their titles.

3) The family members discuss their positive memories or experiences in turn.

4) As a family member tells his/her account, others take notes of the positive points they can find in the person who is talking.

5) Then, each speaker listens to others who are talking about the positive points they have identified in him/her.

6) In the next stage, the members give their lists of positive points to each of the family members in turn. The following lines are some samples of the positive points about a speaker, listed by the group members participating in such a task:

An honest and loyal friend; she speaks clearly and fluently, is independent, has good faith, is a good coordinator, is encouraging, is physically strong, is a true believer, provides good leadership, is creative, helpful, flexible and conforming, practices religion, learns new things easily, is clever, patient and tolerates severe pain, listens carefully, paints well, speaks honestly, is peaceful, is brave, notices the impact of prayers in practice.

7) The positive points are ranked on the basis of their importance.

8) Five to eight points of strength which are called the reliable points are selected from the collected lists of the positive points.

Through this technique, clients developed a new awareness about themselves according to the comments they received from their family members. They were instructed to review their personal memories by combining good and praiseworthy feelings. They then selected the most significant ones and extracted their points of strength. They were able to use the comments of family members for clarifying and sorting their own list of points of strength. They were helped to obtain a more integrated self image through this technique and further developed and maintained it throughout their sessions with the help and support of their family members.

\section{Findings}

Participant 1: Mr. H. F. H. 45 years old, engineer, married to a 40-year-old woman, high school education, 2 daughters who were 13 and 19 years old and a 8-year-old son.

In the first session of psychotherapy, the participant said: "It has been about one year that I am always restless and anxious. I feel a deep sadness and disappointment, always think about death and also have severe backache which specialists believe has a psychological source instead of a physiological one.”

Figure 1 shows the participant's high score in HS (11), demonstrating that he was very concerned about his physical condition and inclined to get attention through physical pains, and his high score in D (12), implying his severe depression. Also, his high score in Hy (15) showed his physical problems.

Dotted lines in Figure 1 demonstrate the participant's profile in MMPI after the intervention. As can be observed, his psychological status after the intervention was within the normal range. The low score in Hs (6) and Hy (10) showed healthy physical situation and the participant's satisfaction with his physical condition and the low score in D (10) implied a healthier mental status and absence of the previously observed depression.

Figure 2 shows high score in physical complaints index (27) before the intervention which revealed his dissatisfaction and concern about his physical condition. The high scores in obsession-compulsion (30), depression (34) and anxiety (30) indexes also indicated the participant's severe anxiety, obsession and depression.

Dotted lines in this figure show the psychological profile of the first participant in SCL-90-R in the posttest stage. As demonstrated by the figure, the participant's psychological and physical state was normal after the intervention. Low score in physical complaint index (8) implied his enhanced physical condition and low scores in obsession-compulsion (9), depres-

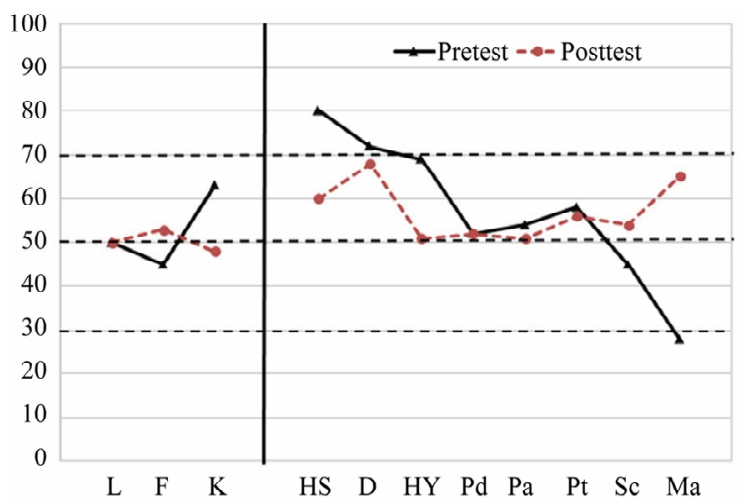

Figure 1.

Psychological profile of the first participant in MMPI in pretest and posttest stages.

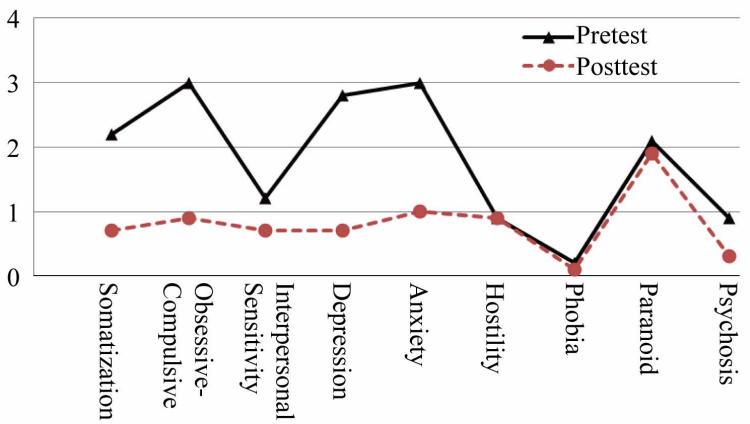

Figure 2.

Psychological profile of the first participant in SCL-90-R in pretest and posttest stages. 
sion (8) and anxiety (10) indexes demonstrated his desirable psychological health.

The comparison of Figures 1 with 2 shows similar findings on MMPI and SCL-90-R before the intervention. The findings of both tests revealed physical problems and dissatisfaction about physical state, depression and anxiety before the intervention.

According to the psychological profile of the first participant in MMPI and SCL-90-R in the pretest and posttest, cognitivebehavioral family therapy was effective in the treatment of psychosomatic disorder (muscular bone) as well as anxiety and depression of the first participant.

Participant 2: Mrs. A. B., 40-year-old, high school education, housewife and married to a 45-year-old man with an M.A. degree and two 13 and 19 year old daughters and one 8-yearold son.

In her first session of psychotherapy, the participant said: "Although we almost understand each other, my husband is a nervous, anxious and depressed man; this has made me anxious, too, and it is about 11 months that I suffer from a severe pain around my knee and lower back. I visited several doctors, but it was useless; they eventually referred me to a psychologist because they thought my physical pains have psychological sources and are caused by high anxiety and stress."

Figure 3 which shows the participant's MMPI profile before the intervention revealed a tendency to exaggerate her undesirable conditions (low scores in $\mathrm{K}$ and $\mathrm{L}$ scales and high scores in F scale). Furthermore, her high score in Hs (11) showed her dissatisfaction with her physical health and that she also tended to receive attention through her physical pains. High scores in Hs and Hy as well as in D scale demonstrated somatization of her inner conflicts and her histrionic traits. The high score in Pd (12) and in Pt (14) demonstrated her paranoia and, her anxiety, fear, restlessness and obsession, respectively.

Dotted lines show a normal posttest profile. The low score in Hs (6) and in Hy (10) implied satisfaction with physical status. The low score in Pd (7) and Pt (8) showed a healthier psychological condition and decrease in her anxiety and paranoia, respectively.

Figure 4 shows the SCL-90-R profile of the second participant before and after the intervention. Before the intervention, her high scores in physical complaint (30), obsession-compulsion (16), depression (18) and anxiety (19) indexes revealed her dissatisfaction with her physical condition as well as sever anxiety, obsession and depression. Dotted lines in the same figure show her posttest profile, indicating that her psychological and physical status was normal after intervention. The low score in physical complaint index (8) showed her satisfaction with her physical condition. Low scores in other indexes such as obsession-compulsion (9), depression (8) and anxiety (10) demonstrated a healthy mental state.

Comparison of Figures $\mathbf{3}$ and $\mathbf{4}$ show a similarity between the findings of MMPI and SCL-90-R. Both tests showed undesirable physical condition, anxiety, obsession and depression in the second participant as well as a significant decrease in her symptoms after the therapy. According to the profiles of the second participant in MMPI and SCL-90-R tests before and after the intervention, it can be concluded that family therapy based on cognitive-behavioral techniques had an effective role in treating the participant's psychosomatic disorder (muscular-bone) and other related psychological disorders.

Participant 3: Mr. S. D. 38 years old, M.A. degree, married

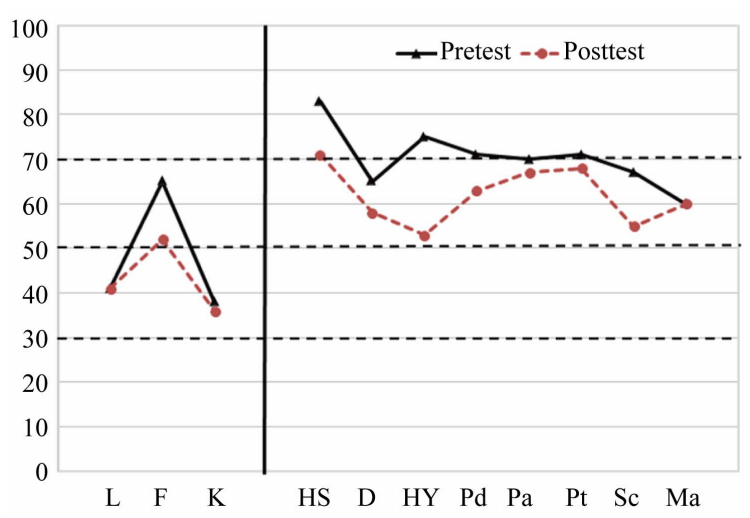

Figure 3.

Psychological profile of the second participant in MMPI in pretest and posttest stages.

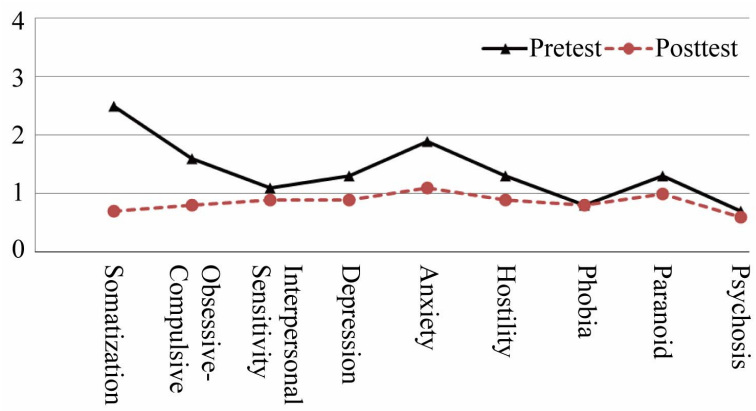

Figure 4.

Psychological profile of the second participant in SCL-90-R in pretest and posttest stages.

to a 28-year-old woman with an MA degree who was a housewife with two sons (7 years and 6 months old).

In his first session of psychotherapy, the participant said: "It has been several years that I have problems with my brother about financial and work issues and this has made me very sensitive and irritable. I have severe anxiety most of the time and it is about 10 months that I am suffering from a severe neck pain and backache. I have visited several physicians, but theysaid that my backache and neck pains are probably caused by psychological stressors."

Figure 5 shows that the participant tried to exaggerate his physical problems (the F score of the participant is higher than his K score) in the pretest stage. The high score in Hs (10) and in Hy (16) also showed the participant's dissatisfaction with his physical conditions and also that he tended to get attention by means of his physical pains. The high score in D (14) implied his isolation and depression. Also, his high score in Pa (10) showed paranoid traits and the high score in Pt (13) expressed his obsession, anxiety and restlessness. Additionally, in Figure $\mathbf{5}$, dotted lines indicated that his psychological status was back to normal in the posttest stage in different scales of MMPI-2. The low scores in Hs (7) and Hy (13) show that the participant's satisfaction with his physical appearance and condition. Also, low scores in D (10), Pa (8) and Pt (11) demonstrated a decrease in other symptoms of isolation, depression, suspicions, anxiety, and restlessness in this subject.

Figure 6 shows that the participant's score in physical complaint (24), obsession (14), depression (29) and anxiety (22) 


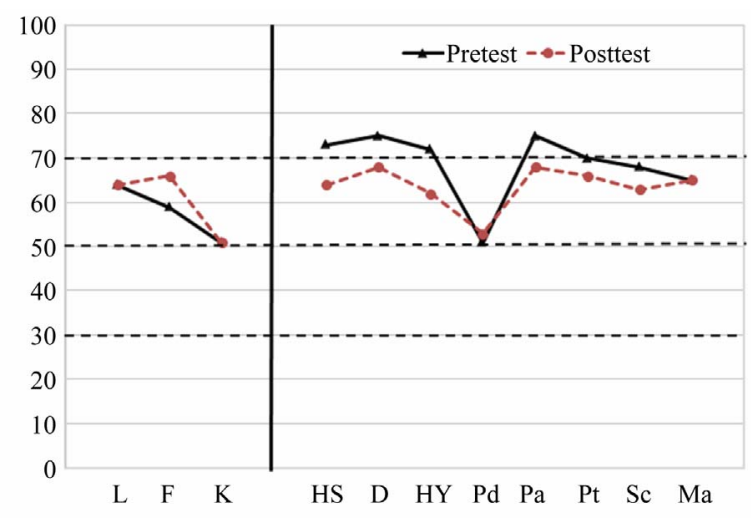

Figure 5.

Psychological profile of the third subject in MMPI-2 in Pretest and Posttest stages.

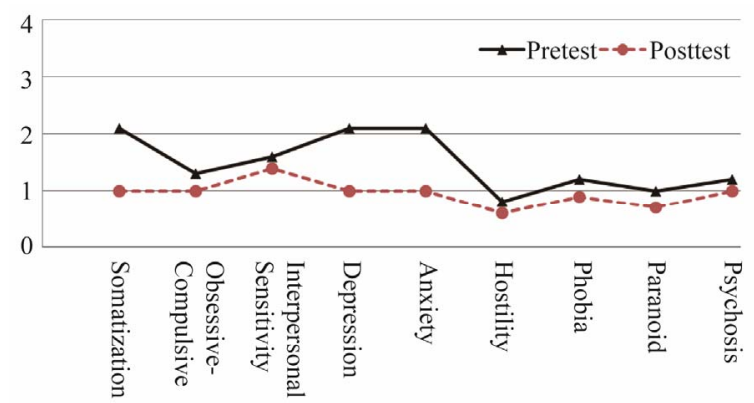

Figure 6.

Psychological profile of the third participant in SCL-90-R in pretest and posttest stages.

indexes were very high in the pretest. Dotted lines in Figure 6 show a decrease in physical complaint (12), obsession (8), depression (9) and anxiety (11) indexes demonstrating a desirable mental and physical state.

Comparison of Figures $\mathbf{5}$ and $\mathbf{6}$ showed high similarity between the findings of MMPI-2 and SCL-90-R before the intervention. The findings of both tests showed physical dissatisfaction, obsession, anxiety and depression in the third participant. Based on the profile of this participant in MMPI-2 and SCL$90-\mathrm{R}$ in the pretest and posttest stages, it can be observed that family interventions based on cognitive-behavioral techniques had an effective role in reducing psychosomatic disorder (muscular-bone) and other related psychological disorders in the third participant.

\section{Discussion}

The findings showed that family interventions based on cognitive-behavioral techniques can be effective in treating psychosomatic disorders (backache-neck pain) and its co morbid psychological disorders such as anxiety, depression, paranoia, obsession and communicative problems. This finding was consistent with the results obtained from the research by Jay et al. (1987), Johansson and Dahl (1988) and Kendal et al. (1997).

Jay et al. (1987) compared cognitive behavioral family therapy with pharmacotherapy and attention control methods in the treatment of bone pains and found that family therapy based on cognitive behavioral techniques was the most efficient therapy in controlling these pains. Johansson and Dahl (1998) studied the effects of cognitive behavioral interventions on a group of individuals who had muscular-bone pains. Their findings revealed that regular cognitive behavioral interventions can successfully treat muscular-bone pains. Kendal et al. (1997) also treated a group of individuals who had anxiety and muscularbone pains with cognitive behavioral techniques.

One of the reasons for the effectiveness of cognitive behavioral family therapy and other related psychological disorders may be related to the nature of psychosomatic problems. Generally, something which is common among people who suffer from psychosomatic problems is their interpersonal problems (such as problems with spouse, parents, siblings, etc.). According to the participants' statements in their psychotherapy sessions, it can be said that this issue was commonly a very prominent problem among the three participants of this study.

Family therapy, therefore, seems quite suitable for these clients since it helps solve the intrapersonal conflicts using techniques such as training problem solving skills, communication skills, assertiveness training, efficient coping skills, positive thinking and cognitive restructuring. This method helped them to not only cope with the existing problems efficiently, but also avoid potential new problems. In family interventions based on cognitive behavioral techniques, there is high emphasis on the modification of negative and damaging communicational means, dysfunctional belief patterns, unreasonable thinking patterns and unreal expectations.

One of the limitations in the present study was its limited number of participants which made the results difficult for generalization. Thus, further similar studies with bigger sample sizes are required. Another suggestion can be to compare this therapeutic method with other therapeutic methods such as individual cognitive behavioral therapy or other modalities and approaches of psychotherapy. Another limitation in this study was concerned with the lack of a control group, which calls for further research on the effectiveness of this method in the treatment of psychosomatic disorders based on the result of this study.

\section{REFERENCES}

American Psychiatric Association (2000). Diagnostic and statistical manual of mental disorders (4th ed.). Washington, DC.

Asmundson, G., Govdony. G, \& Peter, J. (1994). Norton and Felix veloso, Anxiety Sensitivity and fear of pain in patients with recurring headaches. Behavior Research and therapy, 5, 103-113.

Hooman, A. (1997). Standardization of General Health Questionnaire (GHQ) among undergraduate students of Tarbiyat Moallem University. Tehran: Research Institute of Tarbiyat Moallem University.

Johnson, H., \& Dahl, Jo, A. (1998). Effect of a cognitive-behavioral pain management program. Behavior Research and Therapy, 36, 915-930. doi:10.1016/S0005-7967(98)00079-5

Kazdin, A. E., \& Weisz, J. K. (1998). Identifying and developing empirically supported child and adolescent treatments. Journal of Consulting and Clinical Psychology, 66, 19-36. doi:10.1037/0022-006X.66.1.19

Kendal, P. C., Panichelli-Mindel, S. M., Sugarman, A., \& Callahan, S. A. (1997). Exposure to child anxiety: Theory, research, and practice. Clinical Psychology: Science and Practice, 4, 29-39. doi:10.1111/j.1468-2850.1997.tb00096.x

Khodayarifard, M. (2000). Application of positive thinking in psychotherapy from islamic perspective. Journal of Psychology and Education, 5, 141-164.

Knapp, P. H. (1985). Current theoretical concepts in psychosomatic medicine. In H. I. Kaplan, \& B. J. Sadock (Eds.), Comprehensive Textbook of Psychiatry (4th ed., pp. 1113-1121). Baltimore: Wil- 


\section{KHODAYARIFARD, S. A. ZARDKHANEH}

liams \& Wilkins.

Liebman, D., Minuchin, S., \& Baker, L. (1974). The use of structural family therapy in treatment of intractable asthma. American Journal of Psychiatry, 131, 241-253.

Lipowski, Z. J. (1985). Psychosomatic medicine and Liaison Psychiatry. New York: Plenum Press. doi:10.1007/978-1-4613-2509-3

Marnat, G. G. (1990). The manual of mental assessment. New York: Plenum Press.

Mirzaie, R. (1980). The Study of psychometric properties of Symptom CheckList-90 (SCL-90). Unpublished master's thesis, Tehran: University of Tehran.

Najarian, B., \& Davoodi, I. (2001). Preparation and validation of
SCL-25 (Short form of SCL-90-R). Journal of Psychology, 18, 250261.

Peseschkian, N. (1986). Positive family therapy: The family as therapist. Berlin: Springer-Verlag. doi:10.1007/978-3-642-70680-6

Stierlin, H. (1977). Von der psychoanalyses zur Familientherapie. Stuttgart.

Walker, C. E., \& Roberts, M. C. (2001). Handbook of clinical child psychology (3rd ed.). New York: John Wiley \& Sons, INC.

Wolman, B. B. (1988). Psychosomatic disorders. New York: Plenum Publishing Co. doi:10.1007/978-1-4684-5520-5

Woodham, A. (2000). Encyclopedia of natural healing. St. Leonards, NSW: Dorling Kindersley. 